\title{
Hipertermia maligna: A propósito de un caso
}

\author{
CAROLA GUZMÁN H. ${ }^{1}$, ISIDORA ALEUANLLI' ${ }^{2}$ MANUEL GALLEGOS²
}

\begin{abstract}
Malignant hyperthermia $(\mathrm{MH})$ is a rare neuromuscular hereditary disorder, triggered in susceptible individuals by exposure to inhalational agents or succinylcholine and manifested as a hypermetabolic state. We report the case of a 22 years old male patient anesthetized with Desflurane in whom MH was suspected because of unexplained increased levels of End-Tidal carbon dioxide. Dantrolene was administered with good response. Respiratory acidosis and hyperkalemia were also detected but could be easily controlled. The postoperative period was uneventful with the exception of a superficial venous thrombosis at the dantrolene's injection site. The patient was discharged eight days after the episode without sequela. Successful management of a malignant hyperthemia episode must include: early suspicion, asking for help and early treatment with dantrolene.
\end{abstract}

\section{Resumen}

La Hipertermia Maligna es un trastorno neuromuscular hereditario infrecuente, manifestado por un estado hipermetabólico desencadenado en individuos susceptibles por la exposición a anestésicos halogenados o Succinilcolina. Se reporta el caso de un hombre de 22 años anestesiado con Desflurano, en quien el diagnóstico de Hipertermia Maligna se sospechó por la presencia de elevación inexplicada del $\mathrm{CO}_{2}$ espirado, que respondió a la suspensión del Desflurano y administración de Dantroleno. Se observó además hiperkalemia y acidosis respiratoria, que fueron fácilmente compensadas. La evolución postoperatoria fue satisfactoria, a excepción de una trombosis venosa superficial en el sitio de inyección del Dantroleno. El paciente fue dado de alta al $8^{\text {avo }}$ día postoperatorio, sin secuelas. Las claves del manejo exitoso de un episodio de hipertermia maligna están en: Sospecha precoz, solicitar ayuda e inició rápido del tratamiento con Dantroleno.
Key words: Malignant hyperthermia, dantrolene, carbon dioxide, General anesthesia.

Palabras clave: Hipertermia Maligna, dantroleno, dióxido de carbono, anestesia general.

\section{Introducción}

a Hipertermia Maligna (HM) es un trastorno neuromuscular hereditario autosómico dominante, desencadenado por la exposición a anestésicos volátiles halogenados o bloqueadores neuromuscula- res depolarizantes ${ }^{1,2}$

Su incidencia se estima en 1/15.000 anestesias en niños y adolescentes y 1/50.000 -150.000 anestesias en adultos ${ }^{3}$.

La alteración fisiopatológica subyacente, es un defecto del receptor de rianodina en los canales de

\footnotetext{
1 Anestesiólogo Clínica Dávila, Santiago, Chile.

2 Internos Medicina, Universidad de los Andes, Santiago, Chile.

Correspondencia a:

Carola Guzmán

caroliguzmanh@gmail.com
} 
calcio del retículo sarcoplásmico ${ }^{4,5}$. Esto produce una liberación descontrolada de éste catión, lo que desencadena una activación muscular ininterrumpida, manifestada clínicamente como una rigidez generalizada ${ }^{1,2}$.

La contractura muscular mantenida provoca un aumento del consumo de oxígeno $\left(\mathrm{O}_{2}\right)$, incremento en el metabolismo aeróbico y anaeróbico que conduce a exceso de producción de díoxido de carbono $\left(\mathrm{CO}_{2}\right)$, acidosis láctica y aumento de la temperatura corporal².

Clínicamente se observa hipercapnia, taquicardia, rigidez muscular, e hipertermia, siendo este último un signo tardío 1,4,5. También se desarrolla rabdomiolisis, mioglobinemia, mioglobinuria e hiperkalemia por destrucción muscular, lo que puede provocar insuficiencia renal aguda y arritmias cardíacas. En estadios finales de la crisis, sin tratamiento, habrá falla multiorgánica y colapso circulatorio ${ }^{1,2}$.

El tratamiento esencialmente incluye la suspensión de la administración de los agentes desencadenantes y ventilar al paciente con $\mathrm{O}_{2}$ al $100 \%$, esperando una disminución del $\mathrm{CO}_{2}$ espirado $2,4,8,9$. Se debe intentar finalizar el acto quirúrgico lo antes posible. El fármaco específico para tratar las crisis es el Dantroleno sódico, que actúa disminuyendo la liberación de calcio desde el retículo sarcoplásmico ${ }^{1}$.

\section{Caso Clínico}

Un hombre de 22 años, 95 kilos, 1,84 metros de estatura, con diagnóstico de pie cavo aductus bilateral secundario a Neuropatía de Charcot-Marie-Tooth, fue programado para una fasciotomía plantar bilateral electiva. Entre sus antecedentes destacaban hipotiroidismo tratado con Levotiroxina sódica y alergia a la penicilina, además no presentaba historial quirúrgico previo, ni historia familiar de reacción adversa a anestésicos.

Se instaló una vía venosa periférica N²0 G y monitorización básica. Dado que el paciente rehusó recibir anestesia regional, se indujo la anestesia general con 300 mcg de fentanyl, 25 mg de Lidocaína, 200 mg de Propofol, 25 mg de Atracurio y se mantuvo con Desflurano. Se utilizó un tubo endotraqueal $\mathrm{N}^{\circ}$ $8,5 \mathrm{~mm}$ ID y se ventiló con aire y $\mathrm{O}_{2}$ (Fracción inspirada de $\mathrm{O}_{2}$ 50\%). Se administraron $600 \mathrm{mg}$ de Clindamicina y $60 \mathrm{mg}$ de Ketorolaco.

Transcurridos 40 minutos después de la inducción, los valores de $\mathrm{CO}_{2}$ espirado $\left(\mathrm{CO}_{2} \mathrm{ET}\right)$, que se habían mantenido en $30 \mathrm{mmHg}$, comenzaron a aumentar llegando a $60 \mathrm{mmHg}$ en aproximadamente 8 minutos y no mejoraron al incrementar la ventilación; se aumentó la frecuencia respiratoria a 15 respiraciones $\cdot$ minuto $^{-1}$ y el volumen corriente a 800 $\mathrm{ml}$. Se agregó taquicardia (125 lpm), además se detectó que el absorbedor de $\mathrm{CO}_{2}$ aumentó su temperatura al tacto. Ante la sospecha de una crisis de HM se solicitó ayuda a otros anestesiólogos, se suspendió la administración del Desflurano y se inyectó Dantroleno por una vía venosa periférica $N^{\circ} 16 \mathrm{G}$ instalada en la extremidad superior izquierda, en dosis de 2,5 $\mathrm{mg} \cdot \mathrm{kg}^{-1},(240 \mathrm{mg})$. Para la preparación rápida de la dosis necesaria del fármaco se requirieron tres operadores.

Tres anestesiólogos además participaron durante el episodio: Uno se dedicó a iniciar la anestesia total endovenosa con Remifentanil y Propofol. Otro ventiló manualmente al paciente con Ambú y balón de oxígeno al $100 \%$, mientras el tercero cambiaba la maquina de anestesia. Paralelamente se tomó una muestra de sangre arterial que corroboró la hipercapnia, (Tabla

\begin{tabular}{|c|c|c|c|c|c|c|c|c|}
\hline & $\begin{array}{c}\text { Intraop } \\
1\end{array}$ & $\begin{array}{c}\text { Intraop } \\
2\end{array}$ & $\begin{array}{c}\text { Intraop } \\
3\end{array}$ & $\begin{array}{c}\text { Postop } \\
1\end{array}$ & $\begin{array}{c}\text { Postop } \\
2\end{array}$ & $\begin{array}{c}\text { Postop } \\
3\end{array}$ & $\begin{array}{c}\text { Postop } \\
4\end{array}$ & Alta \\
\hline $\mathrm{pH}$ & 7,2 & & 7,31 & & & & & \\
\hline $\mathrm{paCO}_{2}(\mathrm{mmHg})$ & 60 & & & & & & & \\
\hline BE & -3 & & & & & & & \\
\hline $\mathrm{K}^{+}\left(\mathrm{mEq} \cdot \mathrm{L}^{-1}\right)$ & 4,5 & 6,0 & 4,5 & & & & & \\
\hline Creatinina $\left(\mathrm{mg} \cdot \mathrm{dl}^{-1}\right)$ & & & 0,8 & 0,6 & & & 0,7 & \\
\hline Creatinkinasa $U \cdot \mathrm{L}^{-1}$ & 2.297 & & 6.000 & 10.204 & 10.709 & 5.096 & 1.443 & 700 \\
\hline Mioglobinemia $\mathrm{ng} \cdot \mathrm{ml}^{-1}$ & & 5 & 5.797 & & & & & \\
\hline Mioglobinuria & & & + & & & & & \\
\hline
\end{tabular}

Intraop = exámen intraoperatorio; Postop = día del postoperatorio. 
1). Posteriormente se instaló una línea arterial, termómetro esofágico (manteniéndose la temperatura constantemente en $36,2^{\circ} \mathrm{C}$ ) y catéter urinario, recolectándose orinas claras y una diuresis adecuada.

Después de la dosis de carga de Dantroleno se observó una disminución rápida de $\mathrm{ETCO}_{2}$ (a 38 mmHg en 10 minutos), por lo que se decidió no repetir dosis y se inició una infusión de $1 \mathrm{mg} \cdot \mathrm{kg}^{-1} \cdot \mathrm{h}^{-1}$ de Dantroleno por 24 horas, para lo cual se instaló un catéter venoso central.

Se decidió terminar la cirugía y posponer la del otro pié. Al soltar el torniquete de isquemia del muslo se tomaron nuevos exámenes, (Tabla 1, columna INTRAOP2), evidenciandose hiperkalemia. Se inició manejo con $80 \mathrm{ml}$ de suero glucosado al 30\% más insulina cristalina $5 \mathrm{U}$ infundidos en 30 minutos, lográndose una reducción de la kalemia (Tabla 1).

El paciente fue trasladado a la Unidad de Cuidados Intensivos intubado, permaneciendo en ventilación mecánica por 24 horas, sedado con fentanil y propofol. El perfil temporal de los exámenes de laboratorio puede observarse en la Tabla 1.

Cuarenta y ocho horas después de iniciada la crisis, dada su buena evolución, el paciente fue trasladado a Unidad de Cuidados Intermedios. Se solicitó una evaluación neurológica que esbozó dudas respecto al diagnóstico de neuropatía de Charcot-Marie-Tooth, planteándose una posible miopatía.

Al tercer día postoperatorio el paciente refirió dolor y aumento de volumen del miembro superior izquierdo, (Figura 1), diagnosticándose con ecografía doppler una trombosis extensa de sistema venoso superficial asociado a edema reactivo del plano subcutáneo, la cual fue tratada con Rivaroxabán y Vancomicina.

El resto de la evolución postoperatoria fue favorable, siendo dado de alta al octavo día post evento.

\section{Discusión}

La HM es una patología poco frecuente y potencialmente mortal.

El diagnóstico de la HM no es sencillo y existen diversos diagnósticos diferenciales: Sepsis, trastornos tiroideos, feocromocitoma, sobrecalentamiento iatrogénico, mal funcionamiento de la máquina de anestesia, hipoventilación, aumento del ET $\mathrm{CO}_{2}$ por procedimientos laparoscópicos, intoxicación por drogas, síndrome serotoninérgico y síndrome neuroléptico maligno 2,5 . Por esto se han construido escalas con criterios clínicos para objetivar el diagnóstico, existiendo incluso una aplicación para teléfono inteligente

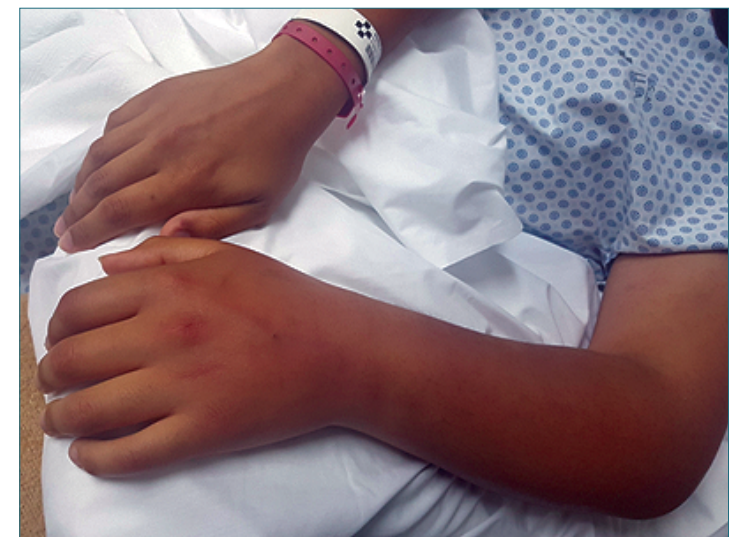

Figura 1. Extremidad superior izquierda del paciente, cursando trombosis venosa superficial.

\section{$\left(\mathrm{MHApp}^{\circledR}\right)^{5,6}$}

En la Tabla 2 se aprecia la escala de clasificación clínica de Bandschapp ${ }^{6}$, que otorga puntaje según criterios clínicos; rango 0-2: Casi nunca; 3-9: Improbable; 10-19: Poco probable; 20-34: Un poco más probable; 35-49: Muy probable; > 50: Certeza.

El paciente presentó 58 puntos, es decir, alto nivel de certeza. Es importante destacar que los criterios diagnósticos se fueron agregando a lo largo de la evolución del episodio.

El diagnóstico e inicio del tratamiento se realizó ante el aumento del $\mathrm{ETCO}_{2}$ de $60 \mathrm{mmHg}$ (a pesar de aumentar los parametros ventilatorios), taquicardia y utilización de agente gatillante (Desflurano).

Una vez sospechado el cuadro se debe iniciar rápidamente el tratamiento, puesto que cada minuto que pasa agrega complicaciones graves y difíciles de manejar (rabdomiolisis, hiperkalemia, insuficiencia renal) y se empeora el pronóstico. Es importante destacar que no se debe esperar la ocurrencia de hipertermia para confirmar el diagnóstico, puesto que este es un signo tardío.

En este caso se cambió la maquina de anestesia, lo cual no es necesario según diversos autores ${ }^{1,8}$. Eso significó perdida de tiempo y de operadores, las recomendaciones son; descontinuar anestésicos volátiles, ventilar con $\mathrm{O}_{2}$ al $100 \%$ con flujos altos, $\left(10 \mathrm{~L} \cdot \mathrm{min}^{-1}\right)$. No es necesario cambiar el circuito circular ni el absorbedor de $\mathrm{CO}_{2}^{8,9}$.

La Food and Drug Administración (FDA), aprobó recientemente la comercialización de una nueva presentación de Dantroleno; RYANODEX ${ }^{\circledR}$ cuyo frasco contiene $250 \mathrm{mg}$ de este medicamento que se diluyen en $5 \mathrm{ml}$ de agua bidestilada ${ }^{5,9}$, esta presentación facilita la preparación y permite una rápida adminis- 


\begin{tabular}{|c|c|c|c|}
\hline Proceso & Criterio Clínico & Puntos & \\
\hline Rigidez Muscular & $\begin{array}{l}\text { - Rigidez muscular generalizada } \\
\text { - Rigidez del Masetero inducida por Succinilcolina }\end{array}$ & $\begin{array}{l}15 \\
15\end{array}$ & \\
\hline Ruptura Muscular & $\begin{array}{l}\text { - Creatin Kinasa }>20.000 \text { UI después del uso de anestésicos incluidos Succinilcolina } \\
\text { - Creatin Kinasa }>10.000 \text { IU después del uso de anestésicos sin uso de Succinilcolina } \\
\text { - Coloración de Orina (cola), en período perioperatorio } \\
\text { - Mioglobinura }>60 \mu \mathrm{g} / \mathrm{L} \\
\text { - Mioglobinemia }>170 \mu \mathrm{g} / \mathrm{L} \\
\text { - K plasma/sangre/suero }>6 \mathrm{mEq} / \mathrm{L} \text { (en ausencia de falla renal) }\end{array}$ & $\begin{array}{r}15 \\
15 \\
10 \\
5 \\
5 \\
3\end{array}$ & $x$ \\
\hline Hipercapnia & $\begin{array}{l}\text {-ET } \mathrm{CO}_{2}>55 \mathrm{mmHg} \text { o } \mathrm{PaCO}_{2}>60 \mathrm{mmHg} \text { (con apropiada ventilación controlada) } \\
\text { - ET } \mathrm{CO}_{2}>60 \mathrm{mmHg} \circ \mathrm{PaCO}_{2}>65 \mathrm{mmHg} \text { (en ventilación espontánea) } \\
\text { - Taquipnea inesplicada }\end{array}$ & $\begin{array}{l}15 \\
15 \\
10\end{array}$ & $x$ \\
\hline Hipertermia & $\begin{array}{l}\text { - Rápido aumento de temperatura } \\
\text { - Inadecuada temperatura }>38,5^{\circ} \mathrm{C}\end{array}$ & $\begin{array}{l}15 \\
10\end{array}$ & \\
\hline Arritmias & $\begin{array}{l}\text { - Inexplicable Taquicardia Sinusal } \\
\text { - Taquicardia Ventricular o Fibrilacion Ventricular }\end{array}$ & $\begin{array}{l}3 \\
3\end{array}$ & $x$ \\
\hline Historia Familiar & $\begin{array}{l}\text { - Con historia de } \mathrm{HM}^{*} \text { en pariente de primer grado } \\
\text { - Sin historia familiar en pariente de primer grado }\end{array}$ & $\begin{array}{r}15 \\
5\end{array}$ & $x$ \\
\hline Otros & $\begin{array}{l}\text { - Exceso de base }>-8 \mathrm{mEq} / \mathrm{L} \\
\text { - PH }<7,25 \\
\text { - Rápida reversión de los signos de HM con Dantroleno }\end{array}$ & $\begin{array}{r}10 \\
10 \\
5\end{array}$ & $\begin{array}{l}X \\
X\end{array}$ \\
\hline Puntaje & & & 58 \\
\hline
\end{tabular}

*Modificado de: Bandschapp, O., laizzo, P. and Girard, T. (2012). Malignant hyperthermia - Update of diagnostics. Trends Anaesth Crit Care 2012;2:218-23.

tración del fármaco, dado que para la dosis inicial en un adulto de hasta $100 \mathrm{~kg}$ de peso bastaría con un sólo vial. En el medio nacional aún no está disponible y sólo se cuenta con DANTRIUM ${ }^{\circledR}$ de 20 mg que debe diluirse en $60 \mathrm{ml}$ de agua bidestilada.

Setenta y dos horas después del episodio de HM, el paciente presentó una trombosis superficial, en la extremidad utilizada para administrar la dosis inicial de Dantroleno. Se han descrito varias complicaciones trás el uso de ésta droga (flebitis, debilidad muscular transitoria, malestar gastrointestinal, compromiso respiratorio en pacientes con desórdenes musculares, necrosis por extravasación y menos frecuentemente trombosis venosa en el sitio de inyección) $)^{5,9}$.

Es importante señalar que en este caso se estaba usando un torniquete de isquemia que al liberarse puede generar aumento del potasio plasmático y del ácido láctico, disminución de la presión arterial de oxígeno $\left(\mathrm{PaO}_{2}\right)$ y del $\mathrm{pH}$. Estos efectos ocurren en los 30 minutos posteriores al retiro del torniquete. También se puede observar un aumento del $\mathrm{CO}_{2}$ espirado, teniendo un máximo 1-3 minutos desde la liberación de la isquemia, retornando a su basal en aproximadamente 10 a 13 minutos ${ }^{7}$, todos estos signos podrían simular una $\mathrm{HM}$.

Otro antecedente a considerar es que el paciente debe completar su evaluación neurológica para confirmar el diagnóstico de una miopatía, esto sería de gran interés, puesto que se ha sugerido una asociación entre HM y ciertas miopatías 5 .

Para finalizar, se recalca la importancia de: No esperar a la aparición de todos los signos clínicos de HM, el pedir ayuda a otros anestesiólogos y solicitar personal de apoyo, dado que la etapa inicial del manejo requiere de muchas acciones simultáneas.

Pese a contar con tablas, puntuaciones y aplicaciones tecnológicas, aún el diagnóstico de HM es eminentemente clínico ${ }^{5}$. 


\section{Referencias}

1. Escobar J. Hipertermia maligna. Rev Med Clin Las Condes 2011;22(3):310-5.

2. Schneiderbanger $D$, Johannsen S, Roewer N, Schuster F. Management of malignant hyperthermia: diagnosis and treatment. Ther Clin Risk Manag 2014 May; 10:355-62.

3. Corvetto AM, Heider MR, Cavallieri BS. Hipertermia maligna: ¿cómo estar preparados? Rev Chil Cir 2013;65(3):279-84.

4. Glahn KP, Ellis FR, Halsall PJ, Müller CR, Snoeck MM, Urwyler A et al.; European Malignant Hy- perthermia Group. Recognizing and managing a malignant hyperthermia crisis: guidelines from the European Malignant Hyperthermia Group. Br J Anaesth 2010 Oct;105(4):417-20.

5. Rosenberg H, Pollock N, Schiemann A, Bulger T, Stowell K. Malignant hyperthermia: a review. Orphanet J Rare Dis 2015 Aug;10(1):93-112.

6. Bandschapp O, laizzo P, Girard T. Malignant hyperthermia - Update of diagnostics. Trends Anaesth Crit Care 2012;2(5):218-23.

7. Kumar K, Railton C, Tawfic Q. Tourniquet application during anesthesia: "What we need to know?". J Anaesthesiol Clin Pharmacol 2016 OctDec;32(4):424-30.

8. Comité de Hipertermia Maligna de la Sociedad de Anestesiologia de Chile. Recomendaciones para el manejo de la Hipertermia Maligna y para el manejo del paciente susceptible de Hipertermia Maligna. Rev Chil Anest 2010;39:197-201.

9. Malignant Hiperthermia Association of the United States [homepage on the internet]. New York:www.mhaus.org [cited 2017 April 20]. Available from: http://www.mhaus.org/ 